\title{
Estilo de vida de homens de uma Estratégia de Saúde da Família
}

\section{Lifestyle of men in a Family Health Strategy}

Como citar este artigo: DIAS, ERNANDES G.; SOUSA, ANILZA A.; MARTINS, CLEONICE C.; CALDEIRA, MAIZA B.

Estilo de vida de homens de uma Estratégia de Saúde da Família. Revista Saúde (Sta. Maria). 2020; 46 (2).

\section{Autor correspondente:}

Nome: Ernandes Gonçalves Dias

E-mail: ernandesgdias@yahoo.com.br Telefone: (38) 99234-7887

Formação Profissional: Mestre em

Ciências pela Universidade de

São Paulo (USP), Ribeirão Preto, SP, Brasil.

Filiação Institucional: Faculdade Verde Norte (Favenorte)

Endereço para correspondência:

Avenida José Alves Miranda,

Bairro: Alto São João

Cidade: Mato Verde

Estado: Minas Gerais

CEP: 39527-000

Data de Submissão:

20/05/2020

Data de aceite:

25/07/2020

Conflito de Interesse: Não há conflito de interesse

\section{(cc) $\mathrm{B} Y-\mathrm{NC}-\mathrm{ND}$}

\author{
Ernandes Gonçalves Dias, Anilza Alves de Sousa, \\ Cleonice Cardoso Martins, Maiza Barbosa Caldeira
}

\section{RESUMO}

Objetivo: Analisar o estilo de vida de homens de uma Estratégia de Saúde da Família de uma cidade do norte de Minas Gerais. Método: Trata-se de um estudo descritivo de abordagem qualitativa realizado com 14 homens com idade entre 27 e 57 anos. Os dados foram coletados no mês de agosto de 2019, no domicílio do usuário através de uma entrevista semiestruturada e analisados mediante Análise do Conteúdo. Resultados: os homens têm um estilo de vida saudável influenciado pela família. Realizam atividade física, têm alimentação saudável e evitam o tabagismo, no entanto o trabalho exerce forte influência sobre o estilo de vida adotado pelos homens. Conclusão: Recomenda-se que os serviços de saúde utilizem de estratégias educativas de promoção da saúde e prevenção de doenças que considerem o contexto e interesse dos homens, visto que a educação em longo prazo pode ocasionar modificação da cultura e melhorar os hábitos de vida da população masculina.

PALAVRAS-CHAVE: Estilo de vida; Saúde do homem; Estratégia Saúde da Família.

\section{ABSTRACT}

Objective: To analyze the lifestyle of men in a Family Health Strategy in a city in the north of Minas Gerais. Method: This is a descriptive study with a qualitative approach carried out with 14 men aged between 27 and 57 years. Data were collected in August 2019, at the user's home through a semistructured interview and analyzed using Content Analysis. Results: men have a healthy lifestyle influenced by the family. They perform physical activity, eat healthy and avoid smoking, however work has a strong influence on the lifestyle adopted by men. Conclusion: it is recommended that health services use educational strategies to promote health and prevent diseases that take into account the context and interest of men, since long-term education can cause a change in culture and improve the lifestyle of women male population.

KEYWORDS: Life style; Men's health; Family Health Strategy. 


\section{INTRODUÇÃO}

O estilo de vida singularmente abarca hábitos, normas e valores demonstrados pelos indivíduos ${ }^{1}$. Estilos de vida saudáveis, no entanto, são apontados como a adesão a um conjunto de comportamentos individuais para favorecer a preservação da saúde².

De modo geral a população masculina tem resistência em relação à adoção de cuidados com a saúde ${ }^{3}$. Essa resistência cresce não somente a sobrecarga financeira sobre a sociedade, como também, o sofrimento físico e emocional do homem, este último associado à cultura e aos estereótipos de gênero, como por exemplo, de que homem não adoece4 .

Porém, sentimentos como medo e vergonha, e causas comportamentais como descuido e outras prioridades de vida ${ }^{3}$ são questões que, de algum modo, afastam o homem do autocuidado e de um estilo de vida mais adequado, à luz das recomendações dos órgãos de saúde.

Nesse sentido, o Ministério da Saúde implementou a Política Nacional de Atenção Integral à Saúde do Homem (PNAISH) que tem como objetivo promover a saúde e prevenir doenças do público masculino ${ }^{5}$. Visto que, um estilo de vida não saudável está associado à obesidade, tabagismo, consumo de álcool, a falta de atividade física e alimentação não saudável, propício a várias doenças, o que tem levado a uma grande quantidade de casos e mortes que poderiam ser evitadas pela eliminação ou redução dos fatores de risco presentes e relacionados ao estilo de vida ${ }^{6}$.

Nesse sentido, é fundamental que os homens compreendam que o adoecimento não está relacionado apenas à presença de sinais e sintomas, mas ao estilo de vida adotado. 0 não reconhecimento da importância do estilo de vida para manutenção da saúde faz os homens se exporem a agravos derivados das concepções de gênero e deve ser um dos focos das ações de saúde voltadas para estes indivíduos ${ }^{3}$.

Isto posto, é essencial que a pessoa seja o centro de qualquer mudança em sua vida e em sua saúde, pois é quem mais conhece sua situação e sabe o que precisa para se sentir bem. Assim, a PNAISH fomenta ações que promovem o autocuidado na população masculina com incentivo para observar seu corpo, mente, ações e formas de cuidar da saúde ${ }^{7}$.

Destarte o exposto faz-se relevante investigar o estilo de vida adotado pelos homens, para melhor compreender seu comportamento de forma que ao compreendê-los, seja possível propor práticas que possam contribuir para promover a saúde e melhorar a qualidade de vida desses sujeitos. Dessa forma, este estudo teve como objetivo analisar o estilo de vida de homens de uma Estratégia de Saúde da Família de uma cidade do norte de Minas Gerais, Brasil.

\section{MÉTODO}

Trata-se de um estudo descritivo de abordagem qualitativa realizado com 14 homens residentes na área de abrangência da ESF A Saúde Vai Até Sua Casa em Catuti, Minas Gerais, Brasil. Para seleção do participante na pesquisa 
o usuário foi abordado ao procurar a ESF e esclarecido sobre a pesquisa e então solicitada sua participação. A busca por participantes aconteceu em dias intercalados durante o mês de agosto no turno matutino com realização de entrevista no domicílio do usuário em dia e horário previamente agendado até que se obteve saturação nos discursos.

Para serem considerados elegíveis os usuários atenderam aos seguintes critérios: ser do sexo masculino; ter idade entre 25 e 59 anos e funções cognitivas preservadas para responder as perguntas; e ser residente na área de atuação da ESF em estudo. A definição desta faixa etária é condizente com o recorte etário estabelecido na PNAISH de 2008. Considerou-se exclusos homens que por ventura procuraram atendimento na ESF em estudo, mas que fosse cadastrado em outra ESF.

Os procedimentos metodológicos deste estudo obedeceram às recomendações da Resolução 466/2012, do Conselho Nacional de Saúde. O projeto de pesquisa foi aprovado pelo Comitê de Ética em Pesquisa (CEP) da Universidade Estadual de Montes Claros (Unimontes) pelo Parecer Consubstanciado 3.513.844, CAAE 16404119.1.0000.5146. Antes da aplicação das entrevistas os participantes foram esclarecidos sobre a pesquisa e assinaram o Termo de Consentimento Livre e Esclarecido.

A captura do empírico foi realizada a partir de uma entrevista de roteiro semiestruturado, elaborada pelos pesquisadores e aplicada individualmente aos participantes. O roteiro de entrevista foi composto por questões norteadoras: você considera seu estilo de vida adequado? Considerando uma semana típica, como são seus hábitos alimentares, de lazer, atividade física, trabalho e descanso? De que maneira seu estilo de vida te expõe a risco?

As entrevistas foram gravadas em áudio por um aplicativo de voz e, posteriormente, transcritas na íntegra para a realização da organização e análise dos dados. Os dados resultantes das entrevistas foram analisados mediante "Análise do Conteúdo" na perspectiva de Minayo ${ }^{8}$. Para resguardar a identidade dos entrevistados, seus nomes foram substituídos por pseudônimos acompanhados de suas respectivas idades, na apresentação do conteúdo.

\section{RESULTADOS E DISCUSSÃO}

O estudo foi realizado com 14 homens, os participantes tinham entre 27 a 57 anos de idade, seis trabalhavam como servidor público municipal, outros seis eram autônomos e dois atendentes de supermercado. Doze eram casados e os demais viviam em união estável. Em relação à escolaridade três eram analfabetos, dois possuíam ensino fundamental completo, outros dois, ensino fundamental incompleto, quatro possuíam ensino médio completo e três concluíram o ensino superior. 


\section{Estilo de vida dos homens}

Os homens utilizam de diversos meios para manter o estilo de vida, frisaram controle da alimentação, lazer, repouso e proteção contra exposição a riscos relacionados ao tabagismo, alcoolismo e realização de atividades expostas ao sol. Quanto à alimentação aparece em seus relatos preocupação em ingerir alimentos saudáveis e tradicionais da alimentação do brasileiro numa frequência entre três e quatro refeições ao dia.

Procuro me alimentar bem, como frutas e verduras, [...] alimentação balanceada, arroz, feijão, frutas e verduras, alimento três a quatro vezes ao dia. (Richard, 42)

Oh, na realidade eu não sou de comer besteiradas. Geralmente é comida básica mesmo, arroz, feijão, bife, uma carne, frutas também né. (Lucas, 38)

No entanto, a jornada de trabalho influencia na frequência com que os homens se alimentam, apesar de reconhecerem que os hábitos alimentares, quando inadequados, interferem na saúde: "[...] teria que melhorar a questão da alimentação, primeira coisa teria que iniciar a questão dos horários, infelizmente por causa do trabalho, não tenho condições de colocar horário pra almoçar." (Adriano, 36). O modo como a jornada de trabalho interfere na alimentação dos sujeitos parece negativo na perspectiva de instauração de uma rotina adequada para se alimentar.

A alimentação deve ser instituída de modo saudável e consciente, visto que se praticada de forma desordenada pode ter como consequência doenças ${ }^{9}$. Hábitos alimentares inadequados podem gerar consequências físicas e psicológicas que afetam a saúde e a qualidade de vida das pessoas. Alimentação desordenada pode levar à obesidade e a doenças carenciais ${ }^{10}$.

Para se manter saudáveis a maioria dos entrevistados procuram praticar atividades físicas regularmente, há relatos de adoção de atividades como a caminhada, futebol e corrida.

Faço atividade física quatro a cinco vezes por semanas, faço caminhada e jogo bola. (João, 46)

[...] futebol, caminhada e corrida para ter boa saúde né. (Richard, 42)

Mais uma vez o trabalho aparece como uma barreira para adoção de hábitos saudáveis, há homens que não praticam atividade física devido a falta de tempo por causa trabalho ou por lesão, que pode estar relacionada, a talvez, prática de atividade física sem a observância de cuidados básicos, como um alongamento antes e após a atividade: "[...] eu jogava bola, mas eu parei, [...] o joelho não deixou mais, o serviço também não deixou mais, mais é o serviço né, porque o horário que eu trabalho não tem como jogar bola." (José Antônio, 37) 
Atualmente as pessoas passam grande parte de seu dia em atividades sedentárias, exemplificadas pelo crescente tempo que se passa sentado, seja no trabalho, no transporte ou no lazer, tal comportamento está associado ao aparecimento de condições crônicas de saúde. Neste contexto, o trabalho é nocivo aos trabalhadores que precisam afastar das atividades físicas por conta da jornada de trabalho ${ }^{11}$. De modo geral a rotina faz com que tanto a alimentação saudável quanto a prática de exercícios físicos caiam no esquecimento, seja por fatores de ordem econômica, social ou pela jornada de trabalho ${ }^{12}$.

Os homens procuram ter sono/repouso satisfatório a suas necessidades individuais de acordo o tempo disponível: "durmo bem, sempre que tenho oportunidade eu repouso, dou uma descansada." (Mário José, 37). No entanto alguns homens relatam ter dificuldade como o sono/repouso em função do trabalho: "[...] às vezes sim, às vezes não, vai depender do tempo de trabalho." (Adriano, 36)

O sono e repouso é um dos principais responsáveis pela recuperação física e mental do organismo e exerce papel importante na homeostasia, cujo desequilíbrio favorece a diminuição da competência imunológica, transtornos mentais, prejuízo no desempenho físico e dificuldades adaptativas. Além disso, pode causar aumento da vulnerabilidade do organismo e colocar a vida em risco, dormir bem possibilita às pessoas terem um bom desempenho em suas atividades diárias ${ }^{13}$.

Em relação ao tabagismo observou-se baixa ocorrência de homens que fumam qualquer tipo de cigarro, isso pode ser reflexo das campanhas antitabagismo, amplamente veiculadas nas mídias, e às ações de promoção da saúde dos serviços de saúde, que tem como consequência a conscientização da população quanto aos riscos, porém, mesmo que em menor frequência, há homens tabagistas que consomem em média cinco cigarros por dia: "[...] fumo uma base de cinco a seis vezes no dia, não fumo a noite só durante o dia." (Mateus, 54)

O consumo de tabaco é um problema global que além de ser prejudicial à saúde, os gastos com tabaco impactam no orçamento doméstico, uma vez que os recursos empenhados com estes produtos poderiam ser direcionados para atender outras necessidades da família ${ }^{14}$. Nesse sentido, a implantação do tratamento de tabagismo na rede básica de saúde certamente contribuiu para queda da prevalência do tabagismo, tanto é que no período de 2006 a 2018 se verificou importante redução nesse indicador. Segundo pesquisa Vigitel, nesse período, registrou-se queda no uso do tabaco por pessoas de 18 a 24 anos de idade (12\% em 2006 e 6,7\%, em 2018), 35 e 44 anos (18,5\% em 2006 e 9,1\% em 2018) e entre 45 a 54 anos (22,6\% em 2006 e 11,1\% em 2018). Além de redução de danos relacionados ao tabagismo a interrupção do uso de tabaco contribui para melhoria na qualidade de vida ${ }^{15}$.

O consumo de bebidas alcoólicas se mostrou inversa em relação ao uso de tabaco, há homens que reconhecem fazer uso de bebidas alcoólicas com maior frequência comparada ao tabagismo, porém o consumo dessas bebidas é, na percepção dos sujeitos, de forma social. 
[...] só social, só fim de semana, não sei certo quantas doses, se eu participar de farra ou churrasco bebo um pouco mais, se não participar bebo oito ou nove doses. (João, 46)

Tomo uma cervejinha todo final de semana, tomo mais de lata, umas duas, quatro, cinco. (Tenório, 37)

O álcool tem sido largamente consumido pela sociedade atual sob a justificativa de se resgatar momentos de prazer a partir do consumo desse tipo de bebida, apesar de serem evidenciados efeitos indesejados ${ }^{16}$. 0 consumo excessivo de qualquer bebida alcóolica, além dos efeitos deletérios sobre a pressão arterial, pode contribuir para 0 excesso de peso que, por sua vez, é fator de risco para a hipertensão ${ }^{17}$.

O lazer aparece nos relatos relacionado ao convívio com outras pessoas para diminuir o estresse. A atividade física, vida sexualmente ativa, participação nos espaços comunitários, como ir à igreja, uso de tecnologias digitais e consumo de bebida alcoólica são itens considerados, pelos homens, como partes integrantes do lazer.

[...] esportes, caminhada, outras atividades como igreja, essas coisas assim sou frequente, vou no terço dos homens, toda quarta. (João, 46)

Tem as coisas que a gente mexe no computador, internet, a gente sai pra algum lugar, toma uma cachaçinha uma cerverjinha, o sexo né. (Tenório, 37)

O combate ao estresse físico, mental e psicológico está aliado à promoção da saúde. As atividades de lazer podem trazer consideráveis benefícios, tanto para saúde física, como para saúde mental e psicológica ${ }^{18}$. Essas atividades são importantes para melhoria da qualidade de vida, entre seus benefícios cita-se o combate ao estresse e promoção do bem-estar que promovem melhora na homeostasia, qualidade da saúde, socialização e interação entre indivíduos ${ }^{19}$.

A família aparece como um suporte para o homem se autocuidar, ela alerta, incentiva quanto à adoção de alimentação saudável e prática de atividade física. O vínculo familiar parece valorizado pelos homens.

[...] sempre me incentivando e alertando, orientando na alimentação, não comer uma comida tão salgada, não comer muita coisa doce nem muito gordurosa [...]. (Marcos, 27)

[...] se vou fazer uma caminhada, tem o grande aqui [filho] que vai também, pega no pé, ele orienta muito, ele gosta de exercício. (Lucas, 38) 
A família oferece ambiente de proteção, abrigo e perseverança à vida e à saúde de seus membros. É o primeiro espaço de cuidado, onde se faz boa parte da assistência inicial e cuidados relacionados à saúde, assim como de orientação e acompanhamento nos momentos difíceis para fornecer apoio e sustentação de maneira efetiva ${ }^{20}$.

O cuidado entre membros da família é balizado pela reciprocidade e se estende pela vida, envolve relações de afeto, atitudes, empatia, respeito e reconhecimento de valores, que, juntos, favorecem as possibilidades de as pessoas cuidarem de si e das outras. Assim, é de fato importante a participação da família para adoção de práticas de autocuidado pelos seus membros ${ }^{21}$.

Apesar dos relatos de comportamentos no sentido de promoção e cuidado com a saúde há homens que reconhecem a exposição a riscos presentes ou relacionados ao trabalho que podem influenciar em seu estado de saúde. Entre eles incluem características do próprio trabalho, a exposição ocupacional a acidentes por animais peçonhentos, agressão por animais bravos, assaltos, ganho de peso e quedas derivadas do trabalho em alturas.

[...] a gente trabalha no comércio, então a qualquer momento você tem algum [assalto], movimenta dinheiro fica complicado. (Mário José, 37)

[...] trabalho com veneno, bichos peçonhentos, trabalho com animais, por exemplo, faz exame com animais de Leishmaniose, o animal pode ser bravo e machucar agente. (Adriano, 36)

[...] queda, peso, risco da altura na instalação de internet e manutenção. (Marcos, 27)

O trabalho é, muitas vezes, visto como um fator desencadeador de modificações das condições de vida dos homens, ele valoriza e dignifica o homem, ao passo que também pode provocar sofrimento e adoecimento quando realizado em condições inadequadas e de exposição a riscos ${ }^{22}$. A percepção dos trabalhadores sobre 0 risco se fundamenta em seus conhecimentos práticos, deduções e informações, assim, é preciso proporcionar aos trabalhadores condições de higiene, redução de riscos, saúde e segurança no ambiente de trabalho. Faz-se importante o uso de EPI para que ele possa executar suas tarefas ${ }^{23}$.

A maioria dos homens entrevistados se expõem excessivamente ao sol, em horários inadequados, sem uso de protetor solar, porém adotam outros meios de proteção, como chapéu, boné e camiseta manga longa.

Nós tomamos muito sol, trabalho de gari, uso boné, chapéu, protetor não.

(Mateus, 54) 
[...] se eu estiver trabalhando, fazendo um bico, servente de pedreiro, aí me exponho [...] uso chapéu, camisa de manga comprida e boné, protetor solar não. (José Antônio, 37)

A inclusão de ações de proteção solar na educação do trabalhador pode contribuir para minimizar os danos cumulativos da exposição à radiação e sua relação com diferentes agravos à saúde. Sabe-se que a exposição solar sem proteção e o conhecimento insuficiente sobre os riscos da exposição são fatores de risco para o aumento no número de casos de câncer de pele e outros danos à saúde ${ }^{24}$.

Dessa forma, recomenda-se o uso de materiais educativos como estratégia para prevenção do câncer de pele em trabalhadores, porém estes materiais devem ser moldados à realidade do trabalhador. Assim, o uso de folhetos informativos é uma forma de se estabelecer aproximação com os trabalhadores e iniciar uma mudança de comportamento 25 .

Além da fotoproteção para prevenção dos efeitos nocivos causado pela radiação ultravioleta é importante destacar a necessidade do uso de vestimentas, acessórios adequados e seguros para exposição ao sol. Os acessórios mais comuns são luvas, óculos escuros, bonés e chapéus ${ }^{26}$.

\section{CONSIDERAÇÕES FINAIS}

O trabalho baliza o estilo de vida dos homens, ele exerce influência sobre a frequência da alimentação, tempo para atividade física e sono/repouso, e expõe o homem a riscos de acidentes de trabalho. Apesar do norte que o trabalho dá ao estilo de vida do homem e ainda que influenciados pela família, há homens que buscam alimentar-se de modo adequado, praticar atividade física e abster-se do tabagismo. Sobretudo de alguma forma conhecem os riscos a que estão expostos e adotam medidas primárias de cuidado.

Nesse sentido, recomenda-se que os serviços de saúde utilizem de estratégias educativas de promoção da saúde e prevenção de doenças que considerem o contexto e interesse dos homens. Isto porque, a educação em longo prazo pode ocasionar modificação da cultura e melhorar os hábitos de vida da população masculina.

\section{REFERÊNCIAS}

1. Almeida MAB, Gutierrez GL, Marques R. Qualidade de vida: definição, conceitos e interfaces com outras áreas de pesquisa. São Paulo: EACH/ USP, 2012, p. 142. 
2. Madeira FB, Filgueira DA, Bosi MLM, Nogueira JAD. Estilos de vida, habitus e promoção da saúde: algumas aproximações. Saúde Soc., 2018;27(1):106-115. [citado em: 17 maio 2020]. Disponível em: https://www.scielo. br/pdf/sausoc/v27n1/1984-0470-sausoc-27-01-106.pdf.

3. Teixeira DBS, Cruz SPL. Atenção à saúde do homem: análise da sua resistência na procura dos serviços de saúde. Rev Cubana Enferm [Internet]. 2016;32(4):[aprox. 0 p.]. [citado em: 19 maio 2020]. Disponível em: http://www. revenfermeria.sld.cu/index.php/enf/article/view/985.

4. Maia SMA, Malagutti W. As dificuldades de percepção do enfermeiro da atenção primária à saúde do homem. Revista HUPE, 2016;15(4):336-342. [citado em: 08 maio 2019]. Disponível em: http://dx.doi.org/10.12957/rhupe.2016.3161.

5. Brasil. Ministério da Saúde. Secretaria de Atenção à Saúde. Departamento de Ações Programáticas e Estratégicas. Política Nacional de Atenção Integral à Saúde do Homem: princípios e diretrizes - Brasília, 2009. 92p.

6. Rezende LFM, Lee DH, Louzada MLC, Canção M, Giovannucci E, Eluf-Neto J. Proporção de casos de câncer e mortes atribuíveis a fatores de risco no estilo de vida no Brasil. Epidemiologia do Câncer, 2019;59:148-157. [citado em: 13 nov. 2019]. Disponível em: https://doi.org/10.1016/j.canep.2019.01.021.

7. Brasil. Ministério da Saúde. Secretaria-Executiva. Secretaria de Atenção à Saúde. Glossário temático: Saúde do homem. Brasília: Ministério da Saúde, 2018. 140p.

8. Minayo MCS. Análise qualitativa: teoria, etapas e confiabilidade. Ciênc. saúde coletiva, 2013;17(3):621626. [citado em: 28 out. 2019]. Disponível em: http://dx.doi.org/10.1590/S1413-81232012000300007.

9. Silva LWP. Alimentação e Percepção. Saber Humano. Cadernos de Ontopsicologia, 2017;ed. esp.:134144. [citado em: 14 out. 2019]. Disponível em: https://doi.org/10.18815/sh.2017v0n0.183.

10. Silva GMF, Silva TKR, Melhem ARF, Tortorella CCS. Avaliação Nutricional, Consumo Alimentar e Percepção de Hábitos Saudáveis entre Escolares de 10 a 14 Anos. Rev. Aten. Saúde, 2018;16(57):30-36. [citado em: 22 nov. 2019]. Disponível em: https://doi.org/10.13037/ras.vol16n57.5215. 
11. Delfino LD. Fatores associados ao padrão de comportamento sedentário em professores da rede pública de ensino. [Dissertação]. Programa de Pós-Graduação em Fisioterapia. Universidade Estadual Paulista. Presidente Prudente, 2018.

12. Flores TR, Gomes AP, Soares ALG, Nunes BP, Assunção MCF, Gonçalves H. Counseling by health professionals and healthy behaviors among the elderly: population-based study in Pelotas, South of Brazil, 2014. Epidemiol. Serv. Saude, 2018;27(1):e201720112. [citado em: 25 nov. 2019]. Disponível em: https://doi.org/10.5123/s167949742018000100012.

13. Godnho MR, Ferreira AP, Santos ASP, Rocha FSA. Fatores associados à qualidade do sono dos trabaIhadores técnico-administrativos em educação de uma universidade pública. Rev Med Saude, 2017;6(3):303-320.

14. Bazotti A, Finokiet M, Conti IL, França MTA, Waquil PD. Smoking and poverty in Brazil: an analysis of the profile of the smoking population based on the 2008-09 Brazilian government Family Budget Survey. Ciência \& Saúde Coletiva, 2016;21(1):45-52. [citado em: 14 out. 2019]. Disponível em: https://doi.org/10.1590/1413-81232015211.16802014.

15. Brasil. Ministério da Saúde. Portal do Governo Brasileiro. Brasil reduz hábito de fumar em $40 \%$ e mantém tendência de queda. Maio de 2019. [citado em: 24 out. 2019]. Disponível em: http://www.saude.gov.br/noticias/agencia-saude/45488-apenas-9-3-dos-brasileiros-ainda-tem-o-habito-de-fumar.

16. Sousa KPA, Medeiros ED, Araújo LF, Belo RP. Representações sociais do álcool entre estudantes universitárias brasileiras. Psicología, Conocimiento y Sociedad, 2019;9(1):205-228.

17. Mussi FC, Portela PP, Barretto LES, Gama GGG, Mendes AS, Macêdo TTS. Consumo de bebida alcoólica e tabagismo em Homens hipertensos. Rev baiana enferm, 2018;32: e20383. [citado em: 06 out. 2019]. Disponível em: http://dx.doi.org/10.18471/rbe.v32.20383.

18. Jacques JPB, Ribeiro RP, Scholze AR, Galdino MJQ, Martins JT. Wellness room as a strategy to reduce occupational stress: quasi-experimental study. Rev Bras Enferm., 2018;71(supl 1):524-31. [citado em: 05 nov. 2019]. Disponível em: http://dx.doi.org/10.1590/0034-7167-2017-0572. 
19. Werle V. Relações entre lazer e saúde em tempos de cultura somática. Revista Brasileira de Estudos do Lazer, 2018;5(2):20-32.

20. Brusamarello T, Maftum MA, Alcantara CB, Capistrano FC, Pagliace AGS. Famílias no cuidado à saúde de pessoas com transtorno mental: reflexos do modelo de assistência. Revista Saúde e Pesquisa, 2017;10(3):441-449.

21. Souza IP, Araújo LFS, Bellato R. Gift and care during the time lived with the family. res.: fundam. care., 2017:9(4):990-998. [citado em: 21 nov. 2019]. Disponível em: http://dx.doi.org/10.9789/2175-5361.2017.v9i4.990-998.

22. Santos SVM, Macedo FRM, Silva LA, Resck ZMR, Nogueira DA, Terra FS. Work accidents and self-esteem of nursing professional in hospital settings. Rev. Latino-Am. Enfermagem, 2017;25:e2872. [citado em: 21 out. 2019]. Disponível em: http://dx.doi.org/10.1590/1518-8345.1632.2872.

23. Miranda AL, Jesus LF, Moreira MFR, Oliveira SS. Percepção de risco: estudo com trabalhadores de um estaleiro expostos a metais. Cad. Saúde Colet., 2019;27(1):93-99. [citado em: 10 nov. 2019]. Disponível em: http://dx.doi. org/10.1590/1414-462x201900010330.

24. Urasaki MBM, Murad MM, Silva MT, Maekawa TA, Zonta GMA. Práticas de exposição e proteção solar de jovens universitários. Rev Bras Enferm., 2016;69(1):126-33. [citado em: 13 nov. 2019]. Disponível em: http://dx.doi. org/10.1590/0034-7167.2016690117i.

25. Nascimento NI, Bardaquim VA, Faria DL, Dias EG, Robazzi MLCC. A experiência da elaboração de um material didático sobre câncer de pele para trabalhadores rurais. Saúde em Redes, 2018;4(3):143-152.

26. Almeida MCS, Barros VGB, Baptista PCP, Silva A. Factors associated with use of personal proactive equipment among community health agents in a coastal municipality in northern São Paulo, Brazil. Rev Bras Med Trab., 2018;16(3):346-52. [citado em: 21 out. 2019]. Disponível em: http://dx.doi.org/10.5327/Z1679443520180134. 